\title{
Benutzungsanleitung I
}

\section{Benutzungsanleitung}

1. Allgemeines

Die in der Sammlung "Besprechungen von Urteilen, Beschlüssen und Gutachten des Bundesfinanzhofs", herausgegeben von Steuerberater Hans U. Loepelmann, Berlin-Charlottenburg, enthaltenen Besprechungen sind nach dem Datum geordnet. Ferner sind Aktenzeichen und Fundstelle im BStBl. III angegeben.

Zur Unterscheidung von Urteilen, die an demselben Tag ergangen sind, dient die eingeklammerte $\mathrm{r} \ddot{\mathrm{m}} \mathrm{m}$ is $\mathrm{ch} \mathrm{e} \mathrm{Z}$ a h 1 in der Kopfsleiste. Dahinter befindet sich die S eit e $\mathrm{nzahl}$ (arabisch) der Besprechung. Ist ein Urteil mehrfach besprochen worden oder überschreitet eine Besprechung den Umfang von zwei Druckseiten, so sind alle Folgeseiten fortlaufend durchnumeriert.

Es wird empfohlen, der Sammlung kein Blatt zu entnehmen, sondern wie mit einem Buch zu arbeiten. Nur dann besteht die Gewähr, daß die Sammlung immer gebrauchsfertig ist.

\section{Zitierweise}

Die Besprechungen werden unter Angabe des Rezensenten, des Datums der Entscheidung, der römischen Klammerzahl und der Seitenzahl zitiert.

Beis pie l: Vgl. Birkholz in Loepelmann, BFH-Besprechungen, BFH v. 13. 1. 1959 (II) 1.

3. Einordnung der Ergänzungslieferungen

Die Ergänzungsblätter werden nach dem Datum der betreffenden Entscheidung bzw. des Gutachtens in das Grundwerk eingeordnet. Dabei ist wiederum bei gleichem Tag die Reihenfolge der römischen Zahlen und innerhalb eines Urteils die Reihenfolge der Seitenzahlen maßgebend.

Für jeden Jahrgang wird ein Abhefteordner geliefert. 
